KUL-TF-99/42

DAMTP 1999-155

LPTENS.99/45

hep-th/9912049

\title{
Isometric embedding of BPS branes in flat spaces with two times
}

\author{
Laura Andrianopoli ${ }^{a}$, Martijn Derix ${ }^{a}$, Gary W. Gibbons ${ }^{b}$ 凹, \\ Carlos Herdeiro ${ }^{c}$, Alberto Santambrogio ${ }^{a}$ \\ and Antoine Van Proeyen ${ }^{a}$ 追 \\ ${ }^{a}$ Instituut voor Theoretische Fysica, Katholieke Universiteit Leuven, \\ Celestijnenlaan 200D B-3001 Leuven, Belgium \\ ${ }^{b}$ Laboratoire de Physique Théorique de l'Ecole Normale Supérieure, \\ 24 Rue Lhomond, 75231 Paris Cedex 05, France [ \\ ${ }^{c}$ D.A.M.T.P., University of Cambridge, \\ Silver Street, Cambridge CB3 9EW, U.K.
}

\begin{abstract}
We show how non-near horizon $p$-brane theories can be obtained from two embedding constraints in a flat higher-dimensional space with two time directions. In particular, this includes the construction of D3-branes from a flat 12-dimensional action, and $M 2$ - and $M 5$-branes from 13 dimensions. The worldvolume actions are determined by constant forms in the higher dimension, reduced to the usual expressions by Lagrange multipliers. The formulation affords insight into the global aspects of the spacetime geometries and makes contact with recent work on two-time physics.

\footnotetext{
${ }^{1}$ Permanent address DAMTP, University of Cambridge

2 Onderzoeksdirecteur, FWO, Belgium

${ }^{3}$ Unité Mixte de Recherche du Central National de la Recherche Scientifique et de l'Ecole Normale Supérieure
} 


\section{Introduction}

It is well known that the description of an anti-de Sitter $(A d S)$ space in $d$ dimensions is facilitated by embedding it in a flat $(d+1)$-dimensional space with two time directions. The embedding is obtained by one constraint and gives a manifestly $S O(d-1,2)$ symmetric description of the global properties of the geometry. Any choice of coordinates on the $d$ dimensional manifold will break this manifest symmetry.

An even more obvious example of extending the dimension for a better understanding of the geometry is the description of the sphere geometry $S^{d}$ in a $(d+1)$-dimensional flat space.

The two examples above are combined in [1], where the $A d S_{p+2} \times S^{n-1}$ near-horizon geometry of $p$-branes in $D=p+n+1$ dimensions is described starting from a flat $(D+2)$ dimensional space. Two constraints are imposed, which, respectively, reduce $p+3$ dimensions to the $A d S_{p+2}$ manifold and $n$ dimensions to the $S^{n-1}$-sphere. The Born-Infeld actions for the near-horizon theories of various branes are obtained from those in a flat $(D+2)$-dimensional space by adding terms with two Lagrange multipliers, imposing the embedding constraints. The Wess-Zumino (WZ) terms in the actions are obtained from a $(p+2)$-form, which is integrated over a $(p+2)$-dimensional manifold which has the worldvolume as its boundary.

It is interesting therefore to ask whether the full spacetime metric of a brane may be isometrically embedded in $\mathbb{E}^{D, 2}$. Apart from its possible relevance to the existence of exotic theories with two times, it is possible that isometric embeddings of $D$ - and $M$-brane metrics as submanifolds of flat space may have some technical advantages for quantizing particles or strings moving in these backgrounds, since one may think of them as free particles or strings subjected to nonlinear constraints. One might try to implement Dirac's procedure for quantization with constraints.

We will generalize the constructions of [1] for non-near-horizon brane geometries. We will show that even if the geometry is not a product of an $A d S$ with a sphere, the brane geometry can be embedded in a flat $(D+2)$-dimensional space with two time directions. The two constraints are in this case not expressed on separate coordinates of the embedding space, but involve a non-trivial mixing of the coordinates. Also the forms for the WZ terms

are obtained in this picture. For that construction, we follow [3] where it is proposed that a $p$-brane evolving in a space with two times should couple to a $(p+3)$-form field strength. The field strength is contracted to a $(p+2)$-form which can be used for the WZ term. To do this contraction we will have to introduce an extra vector field which will be of an elegant form only in the near-horizon limit.

One may wonder whether the whole geometry cannot be embedded with just one extra dimension and why we need two time directions in the embedding space. First of all it has been shown [4] that the embedding of a surface in a flat space of co-dimension 1 imposes, by 
use of the Einstein equations of motion, that the surface has constant curvature, if the surface has dimensionality $d>2$. This corresponds to the familiar cases of the embedding of spheres and (anti-)de Sitter manifolds in flat spaces with only one more dimension. Therefore, in order to embed a brane background, we need at least two extra dimensions.

To determine the signature of the metric of the embedding space we use the following argument. An interesting aspect of brane spacetimes is that they are not globally hyperbolid According to Penrose [5], a global isometric embedding into a flat space with one time, i.e. into $\mathbb{E}^{n, 1}$, is not possible for a spacetime which is not globally hyperbolic. One needs at least two times. Penrose's argument is essentially that the restriction of the time coordinate $X^{0}$ of $\mathbb{E}^{n, 1}$ to the embedded spacetime $M$ would serve as a time-function on $M$, i.e. a function which increases along every future-directed timelike curve. Moreover, if the embedding is suitably regular, the level sets (constant time slices) would actually serve as Cauchy surfaces on M, implying global hyperbolicity. No such obstruction arises for embeddings into flat spacetimes with more than one time.

What we will describe in this paper is therefore a minimal embedding of general brane backgrounds in flat spaces with two extra dimensions and $(D, 2)$ signature.

In the case of particles in brane backgrounds, the constraints determining the embedding in a space with two time directions can be studied in the Hamiltonian formalism leading to canonical quantization. We first treat these constraints as second-class constraints, finding the corresponding system of Dirac brackets. Furthermore, as discussed in [6, 7], particle systems in spaces with two times can also be associated with first-class constraints closing an $S p(2)$ algebra (the local version of the $S p(2)$ relating coordinates and momenta in phase space). We will obtain these constraints. However, it turns out that the action for a particle in a brane background can be written as a gauge-fixed form of the action constructed in [6] only in the case of a conformally flat metric, i.e. in the near-horizon limit.

In section 2 we give the embedding of the geometry, first in general and then comment on the near-horizon approximation, and on connecting regions separated by coordinate singularities. The worldvolume actions will be constructed in section 3. The essential step in that section is the construction of the forms. First, general results for the electric field strengths are given, before completing the construction for the cases D3, M2 and M5 separately. In section 1 we will give the Dirac analysis of constraints in the particle case and make connection with the works of [6].

\footnotetext{
${ }^{4} \mathrm{~A}$ space is called globally hyperbolic if it possesses a Cauchy surface.
} 


\section{Embedding: The geometry}

In this section we describe the embedding of an $S O(n)$ invariant $p$-brane in a $(D+2)=$ $(n+p+3)$-dimensional spacetime. We will obtain the embedding by demanding that the known metric of the brane be obtained from a flat $(D, 2)$ metric. Thus, we demand that the embedding is isometric. With an ansatz where the $D+2$ flat coordinates are written in terms of a particular mixing of hyperspherical and horospherical coordinates, 3 of these coordinates are left as unknown functions of the coordinate $r$ describing the distance from the brane. This leads to two constraints, as we first show for a rather general type of metric in $D$ dimensions with $S O(p, 1) \times S O(n)$ symmetry. Then we specialize to the metric for non-dilatonic $D$ - and $M$-branes. In the third subsection, we look to the structure beyond the horizon to obtain an insight in the global structure.

\subsection{The general case}

The $p$-brane original spacetime is $D$-dimensional, and the geometry can generally be described by a metric of the form

$$
\mathrm{d} s^{2}=A(r)^{2}\left[-\mathrm{d} t^{2}+\mathrm{d} x_{p} \cdot \mathrm{d} x_{p}\right]+B(r)^{2} \mathrm{~d} r^{2}+C(r)^{2} \mathrm{~d} \Omega_{n-1}^{2},
$$

where $\mathrm{d} x_{p} \cdot \mathrm{d} x_{p}$ is the $p$-dimensional spacelike part on the worldvolume, and $\mathrm{d} \Omega_{n-1}^{2}$ is the $n$-sphere metric. The metric has manifest Poincaré invariance on the worldvolume, as well as $S O(n)$ invariance in the transverse space.

Now we consider the embedding space, for which we consider Cartesian coordinates $X^{M}$, with $M=0, \ldots, D+1$, which we divide as follows:

$$
X^{M}= \begin{cases}X^{\mu} & \mu=0, \ldots, p \\ X^{p+1} & \\ X^{p+2} & \\ X^{\alpha} & \alpha=p+3, \ldots, D+1 .\end{cases}
$$

The flat metric with signature $(D, 2)$ can be written as

$$
\mathrm{d} s^{2}=-\left(\mathrm{d} X^{0}\right)^{2}+\left(\mathrm{d} X^{1}\right)^{2}+\cdots+\left(\mathrm{d} X^{p+1}\right)^{2}-\left(\mathrm{d} X^{p+2}\right)^{2}+\cdots+\left(\mathrm{d} X^{D+1}\right)^{2} .
$$

\footnotetext{
${ }^{5}$ An embedding is said to be isometric at a point $p$ iff $g(U, V)=\overline{g\left(f_{*} U, f_{*} V\right)}$, where $(M, g)$ is the manifold to embed, $(\bar{M}, \bar{g})$ the embedding space, $f$ is the embedding $(f:(M, g) \rightarrow(\bar{M}, \bar{g})), f_{*}$ is the differential of $f$, and $U$ and $V$ are two elements of $T_{p} M$ [8, 9]. This basically means that length and angles are preserved in mapping from $T_{p} M$ to $T_{p} \bar{M}$. When chart-induced bases are used both on $M$ and $\bar{M}$, we have for the isometric condition $g_{\alpha \beta}=X_{{ }_{, \alpha}} X^{B}{ }_{, \alpha} \bar{g}_{A B}$.
} 
From the viewpoint of the embedding, the $(D+2)$-dimensional coordinates $X^{M}$ are the embedding functions. Hence, the $D$-dimensional geometry will be described by two constraints in the $(D+2)$-dimensional coordinates, in the same way that a sphere $S^{2}$ embedded in $\mathbb{R}^{3}$ is described by one constraint in the 3-dimensional coordinates, namely the equation $X^{2}+Y^{2}+Z^{2}=R^{2}$ in Cartesian coordinates. We now describe one way to obtain these two constraints. We start by making a change of coordinates in the $(D+2)$-dimensional spacetime so as to make manifest a subgroup $S O(p, 1) \times S O(n) \subset S O(p+n+1,2)$. This is achieved by using a mixture of hyperspherical and horospherical coordinates $\left\{\rho, z, x^{\mu}, \beta, n^{\alpha}\right\}$, as follows:

$$
\begin{aligned}
& X^{p+2}-X^{p+1}=\frac{\rho}{z} \\
& X^{p+2}+X^{p+1}=\rho z+\frac{\rho}{z} x^{\mu} x_{\mu}, \\
& X^{\mu}=\rho \frac{x^{\mu}}{z} \\
& X^{\alpha}=\beta n^{\alpha},
\end{aligned}
$$

where $n^{\alpha}$ parametrize the sphere $S^{n-1}$. With this change of coordinates, the metric reads

$$
\mathrm{d} s^{2}=-\mathrm{d} \rho^{2}+\frac{\rho^{2}}{z^{2}}\left[\mathrm{~d} x^{\mu} \mathrm{d} x_{\mu}+\mathrm{d} z^{2}\right]+\mathrm{d} \beta^{2}+\beta^{2} \mathrm{~d} n^{\alpha} \mathrm{d} n^{\alpha} .
$$

In the comparison between (2.5) and (2.1) we identify $\mathrm{d} x^{\mu} \mathrm{d} x_{\mu}$ with $-\mathrm{d} t^{2}+\mathrm{d} x_{p} \cdot \mathrm{d} x_{p}$ and $\mathrm{d} n^{\alpha} \mathrm{d} n^{\alpha}$ with $\mathrm{d} \Omega_{n-1}^{2}$. Then $\beta, \rho$ and $z$ are functions of $r$ to be determined. The comparison gives

$$
\begin{aligned}
& \beta=C(r) \\
& \frac{\rho}{z}=A(r) \\
& -\mathrm{d} \rho^{2}+\frac{\rho^{2}}{z^{2}} \mathrm{~d} z^{2}+\mathrm{d} \beta^{2}=B(r)^{2} \mathrm{~d} r^{2} .
\end{aligned}
$$

These encode the two aforementioned constraints, since we reduce from three degrees of freedom $(\rho, z, \beta)$ to one $(r)$. The differential equation can further be written as

$$
\frac{C^{\prime 2}-B^{2}}{A^{\prime}}=(\rho z)^{\prime} \equiv F^{\prime}
$$

\footnotetext{
${ }^{6}$ The $n^{\alpha}$ can be seen as the Cartesian coordinates in $R^{n}$ so that they define the unit sphere $S^{n-1}$ as $\sum_{\alpha}\left(n^{\alpha}\right)^{2}=1$ and hence, the metric on this sphere, $\mathrm{d} \Omega_{n-1}^{2}$ is given by the Euclidean metric on $R^{n}$ restricted to the hyperspherical hypersurface $\Sigma$. The relation between $n^{\alpha}$ and the usual hyperspherical angular coordinates $\left(\theta, \phi_{1}, \ldots, \phi_{n-2}\right)$, is, for example, for $p=3: n^{6}=\sin (\theta) \sin \left(\phi_{1}\right) \sin \left(\phi_{2}\right) \sin \left(\phi_{3}\right) \sin \left(\phi_{4}\right)$, $n^{11}=\sin (\theta) \sin \left(\phi_{1}\right) \sin \left(\phi_{2}\right) \sin \left(\phi_{3}\right) \cos \left(\phi_{4}\right), n^{10}=\sin (\theta) \sin \left(\phi_{1}\right) \sin \left(\phi_{2}\right) \cos \left(\phi_{3}\right), n^{9}=\sin (\theta) \sin \left(\phi_{1}\right) \cos \left(\phi_{2}\right)$, $n^{8}=\sin (\theta) \cos \left(\phi_{1}\right), n^{7}=\cos (\theta)$.
} 
where the prime denotes differentiation with respect to $r$. Hence, we can impose the constraints while making the coordinate transformation (or, in other words, while defining the embedding functions) by replacing (2.4) by

$$
\begin{aligned}
X^{-} & \equiv X^{p+2}-X^{p+1}=A(r) \\
X^{+} & \equiv X^{p+2}+X^{p+1}=F(r)+A(r) x^{\mu} x_{\mu} \\
X^{\mu} & =A(r) x^{\mu} \\
X^{\alpha} & =C(r) n^{\alpha} .
\end{aligned}
$$

We can, furthermore, express the constraints in terms of the $X^{A}$ coordinates only. Denoting the inverse function with an overbar, i.e. $\bar{f}(f)=f(\bar{f})=$ identity, we can write $r=\bar{A}\left(X^{p+2}-X^{p+1}\right)$. Thus, our two constraints are

$$
\begin{aligned}
\phi_{1}\left(X^{+}, X^{-}, X^{\mu}\right) & =X^{-} X^{+}-X^{\mu} X_{\mu}-X^{-} F\left(\bar{A}\left(X^{-}\right)\right)=0 \\
\phi_{2}\left(X^{-}, X^{\alpha}\right) & =\sum_{\alpha}\left(X^{\alpha}\right)^{2}-\left[C\left(\bar{A}\left(X^{-}\right)\right)\right]^{2}=0 .
\end{aligned}
$$

These constraints are thus determined by the functions $A, C$ and $F$. The latter is determined up to a constant by (2.7) in terms of $A, B$ and $C$. Note that so far there is no definition of the radial variable $r$. We can use different parametrizations; for example, it will turn out that in some cases it is useful to take $A$ or $C$ itself as the radial variable. Thus in the first case one may take the equations here with $A(r)=r$, and in the second case $C(r)=r$. In the standard brane cases, the functions $A, B$ and $C$ will take the form of some harmonic function to some power in the transverse space to the brane. We will further adopt the name $r$ for that transverse coordinate, use just the name $A$ for the parameter in the first mentioned parametrization and use $R$ for the radial coordinate such that $C(R)=R$.

\subsection{Non-dilatonic $D$ - and $M$-branes}

From now on we will assume that the functions $A, B$ and $C$ are indeed harmonic functions in $n$ dimensions with a flat limit at $r \rightarrow \infty$. For non-dilatonic $D$ - and $M$-branes, they are of the following form:

$$
\begin{aligned}
H & =\left(1+\frac{1}{r^{\kappa}}\right) \\
A(r) & =H^{-1 /(p+1)} \\
B(r) & =H^{1 / \kappa} \\
C(r) & =r H^{1 / \kappa} \\
\kappa & \equiv n-2=D-p-3 .
\end{aligned}
$$


Here a priori $r>0$ and $r=0$ corresponds to the horizon, but we will come back to this in section 2.3.2. The values for $p, \kappa$ and

$$
w=\frac{p+1}{\kappa}
$$

for these branes are summarized in table [1. Under these conditions, the second constraint

\begin{tabular}{|l|lll|}
\hline & $p$ & $\kappa$ & $w$ \\
\hline D3 & 3 & 4 & 1 \\
$M 2$ & 2 & 6 & $\frac{1}{2}$ \\
$M 5$ & 5 & 3 & 2 \\
\hline
\end{tabular}

Table 1: The non-dilatonic branes

in (2.9) implies

$$
\sum_{\alpha}\left(X^{\alpha}\right)^{2}=\frac{\left(X^{-}\right)^{-2 w}}{\left[\left(X^{-}\right)^{-(p+1)}-1\right]^{2 / \kappa}} .
$$

With an explicit form for the functions $A, B$ and $C$ we can evaluate the function $F$. Using (2.7) we obtain

$$
F^{\prime}(r)=-w r^{1-\kappa}\left(1+r^{-\kappa}\right)^{\frac{2}{\kappa}+\frac{1}{p+1}-1}\left(1+2 r^{\kappa}\right),
$$

which can be integrated to give (up to a constant)

$$
F(r)=-\frac{w}{\kappa}\left[B_{\frac{r \kappa}{r^{\kappa}+1}}\left(\frac{-1}{p+1}, 1-\frac{2}{\kappa}\right)+2 B_{\frac{r \kappa}{r^{\kappa}+1}}\left(\frac{p}{p+1},-\frac{2}{\kappa}\right)\right] .
$$

Here we used the incomplete beta function

$$
B_{x}(a, b)=\int_{0}^{x} t^{a-1}(1-t)^{b-1} \mathrm{~d} t=a^{-1} x_{2}^{a} F_{1}(a, 1-b ; a+1 ; x),
$$

which is defined for $0<x \leq 1$. This means that $F(r)$ is well defined in the region $r>0$, which is what we were looking for.

The near-horizon approximation. It is well known that the isometry group of $\operatorname{Ad} S_{n}$, i.e. $S O(n-1,2)$, acts as the conformal group on an $(n-1)$-dimensional Lorentzian manifold (in particular, on its conformal boundary). It has also been known for some time [10] that 
$A d S$ spacetimes arise as the geometry of some BPS branes in the near-horizon limit. For instance, for the $M 2-, M 5$ - and $D 3$-branes, the near-horizon geometry is $A d S_{p+2} \times S_{D-p-2}$. Therefore, if we study these branes on the backgrounds of their own near-horizon geometries, conformal field theories on the branes worldvolumes should arise. In [1], such a study was performed by embedding the near-horizon supergravity solutions in a $(D+2)$-dimensional spacetime. As we discussed in the previous section, for the three mentioned solutions the geometry takes a form like (2.1) with $A, B$ and $C$ given by (2.10). For small $r$, we obtain

$$
B=\frac{C}{r} \sim \frac{1}{r}, \quad A \sim r^{1 / w}, \quad F \sim w^{2} r^{-1 / w}
$$

so that for the three cases, the embedding functions (2.8) reduce to those used in [1].

\subsection{Passing through the horizon}

Using the embedding (2.8) we can now study the global properties of the brane geometries. Before considering the higher-dimensional $D$ - and $M$-branes, let us first look at the easier and lower-dimensional example of the extreme Reissner-Nordstrøm (RN) black hole (a large list of embedding functions for other solutions of general relativity is given in [1]).

\subsubsection{Example: extreme Reissner-Nordstrøm black hole.}

The RN black hole fits our general embedding scheme with $D=4$ and $p=0, \kappa=1$, $w=1$. Here, rather than working with the radial variable $r$ as in (2.10), we use the variable $R$, which, as mentioned at the end of section 2.1, has the property $C(R)=R$. Then the functions $A$ and $B$ are given by $A(R)=B(R)^{-1}=1-1 / R$. The variable $R$ is just shifted with respect to $r$, as $R \equiv r+1$, and the horizon is now at $R=1$, and $R=0$ corresponds to the singularity. Using (2.7), we then find

$$
F_{R N}(R)=\frac{1}{R-1}-3 R-R^{2}-4 \log |R-1| .
$$

The near-horizon limit of the extreme RN metric is $A d S_{2} \times S^{2}$. AdS spaces are naturally defined by their embedding. Figure 1 shows $A d S_{2}$ using two different parametrizations. 

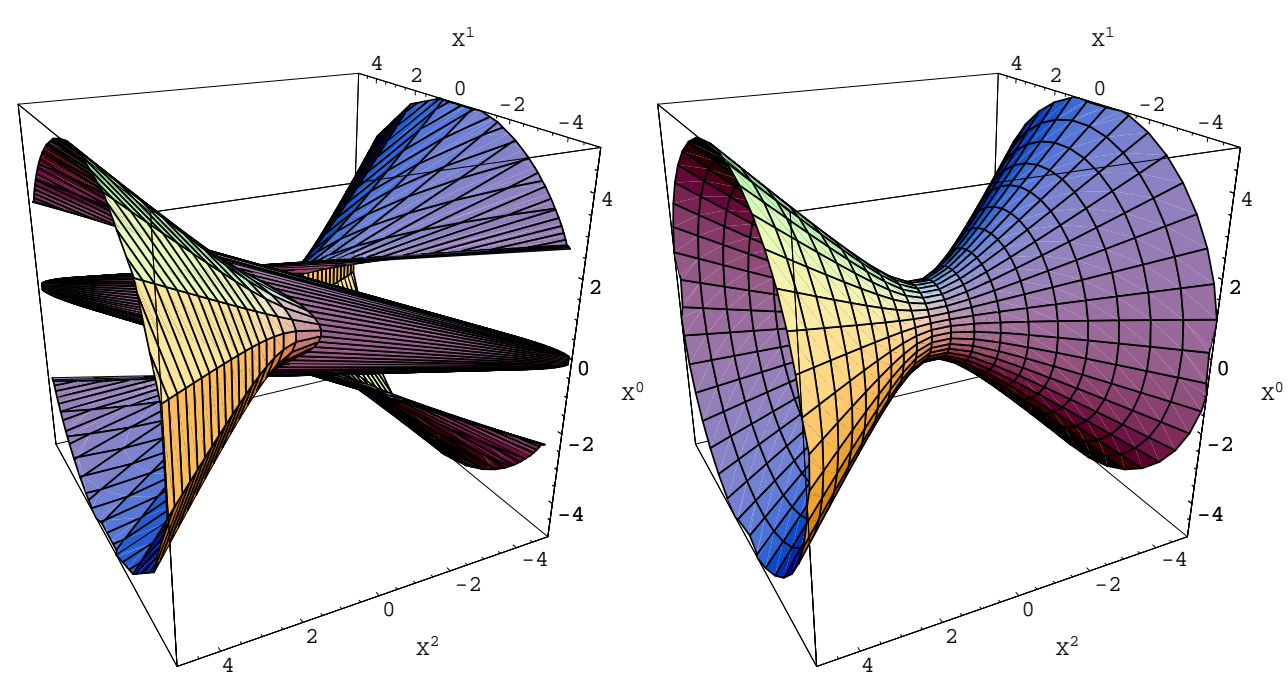

Figure 1: $A d S_{2}$. Left, horospherical coordinates $\left(X^{-}=A, X^{+}=1 / A-A t^{2}, X^{0}=A t\right)$ and right, hyperspherical coordinates $\left(X^{0}=\sqrt{1+u^{2}} \cos v, X^{1}=\sqrt{1+u^{2}} \sin v, X^{2}=u\right)$. Note $X^{ \pm}=X^{1} \pm X^{2}$.

Note that the horospherical embedding seems to have problems at $X^{-}=0$, because this parametrization goes bad as $A=X^{-} \rightarrow 0$. This is just an artefact of the parametrization and is not a feature of the embedded surface itself, as can be seen from the other parametrization. The holes in the left figure are due to limits in the range of $t$ and $r$. For example, we have to start from a finite $r>0$ in order to have no singularities in the functions.

The entire Reissner-Nordstrøm black hole geometry can be drawn in a similar fashion using parametrization (2.8) as is shown in figure 2. Note that we used a slightly different orientation as in the $A d S_{2}$ pictures.

We can read off the following global features from the picture. The geometry consists of 2 distinct regions: region $\mathrm{I}$ is the asymptotically flat region for $R>1$ which corresponds to $X^{-}>0$. For big $R$ the surface flattens and $X^{-} \rightarrow 1$, which is the flat limit. Region II is the region inside the horizon $\left(X^{-}<0\right)$. The singularity $(R=0)$ corresponds to $X^{-} \rightarrow-\infty$. The two regions are connected in an $A d S$ throat (cf figure 1). It seems that these two regions are disconnected, the constant time lines all diverge near $X^{-}=0$ and never cross the horizon. As in the $A d S$ case, this is just an artefact of the parametrization. Actually, we know that the near-horizon geometry is equivalent to $A d S_{2}$, which has no problems at its 'horizon'. 

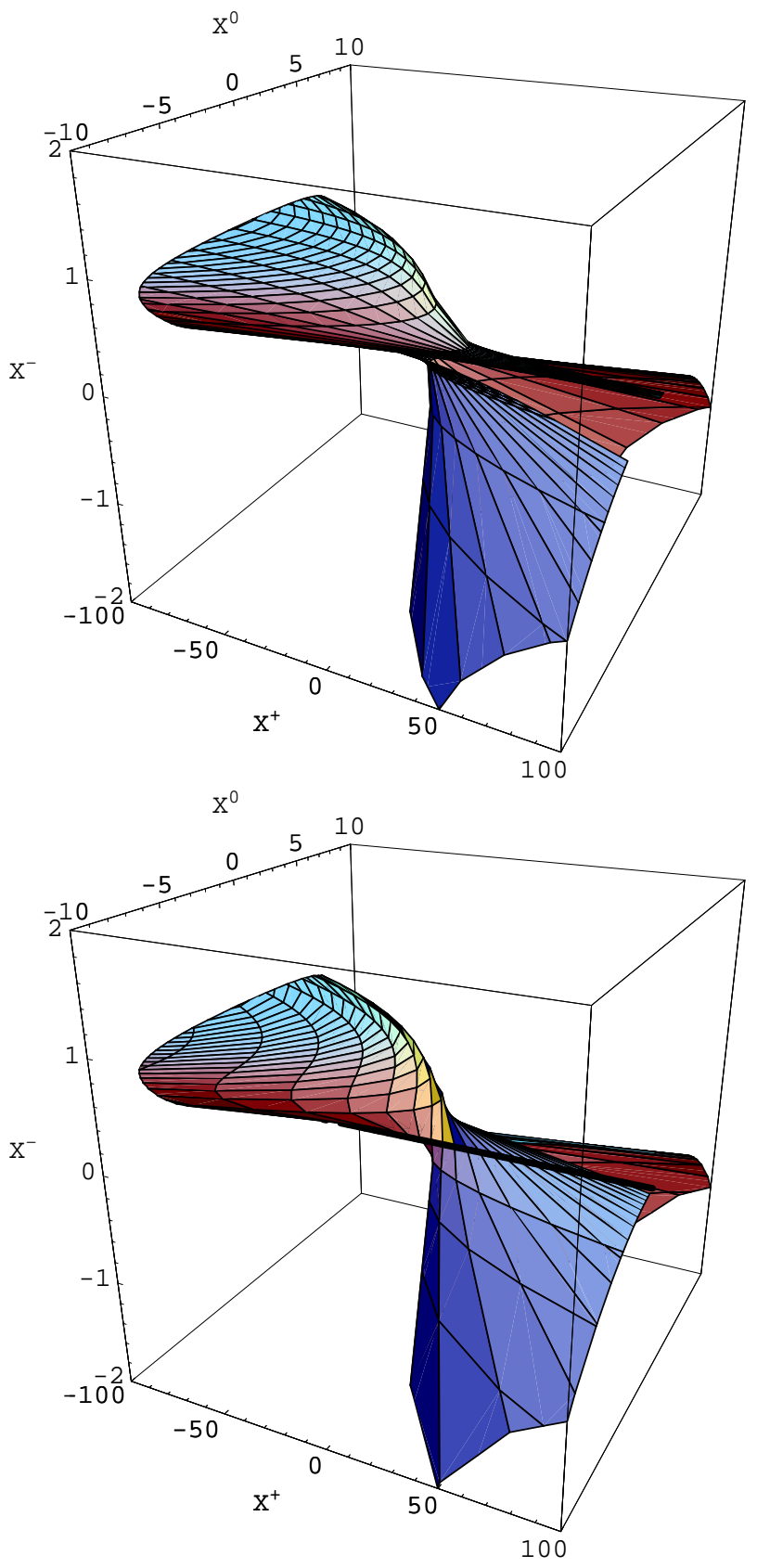

Figure 2: Extreme Reissner-Nordstrøm black hole. Top, parametrized by $R$ and $t$. Bottom, in advanced Finkelstein coordinates $R$ and $v$ (with $v=t-R^{*}$, where $R^{*}=\int \frac{B(R)}{A(R)} \mathrm{d} R$ ). The fat line indicates the horizon. Recognize the two different regions: on the left the asymptotically flat region $I\left(X^{-}>0\right)$ and on the right the interior region $I I\left(X^{-}<0\right)$ connected in the AdS throat. Compare the constant $v$ (bottom) with constant $t$ lines (top) which correspond to infalling lightlike geodesics. In the $v$ parametrization they pass through the horizon into the interior region and end at the singularity instead of diverging at the horizon. 
One of the features of $A d S$ spaces is that they admit closed timelike curves, which can be clearly seen in figure 1. The usual remedy for this is to consider the covering space $C A d S$ instead of $A d S$ itself. Looking at figure 2 we see that our RN black hole geometry suffers from the same problem, it admits closed timelike curves (remember that $X^{0}$ and $X^{2}$ both are timelike directions). Again this is remedied by considering the covering space. The result of this of course is that the space then consists of multiple universes. It is this covering space that is depicted in the familiar Penrose-Carter diagram for the extreme RN black hole (figure 3).

In textbooks one usually shows that the horizon of a black hole is regular by adopting special coordinates in which you can follow the geodesics through the horizon inside the black hole, the so-called advanced (or retarded) Finkelstein coordinates. We can do the same thing with our embedding as is shown in figure 2 .
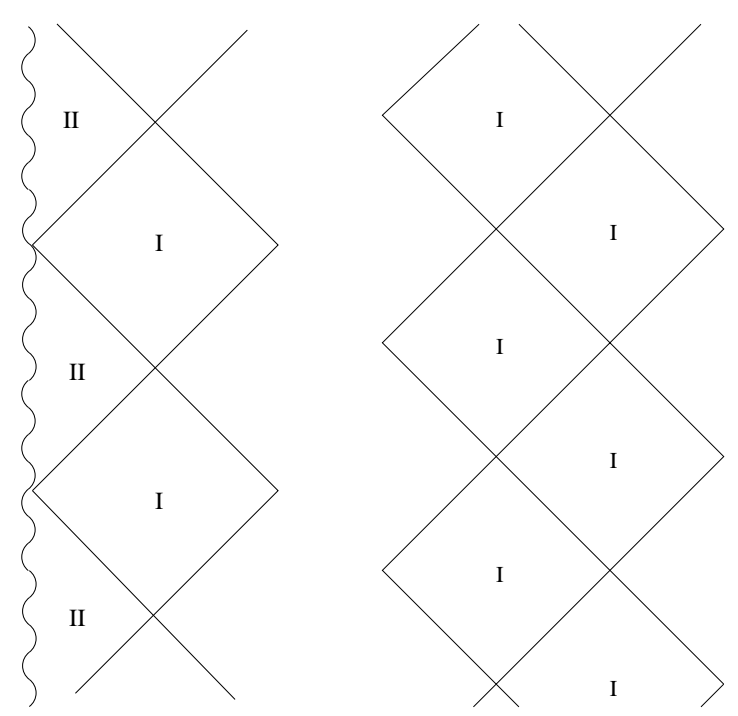

Figure 3: Penrose-Carter diagrams. Left, $p$ even (including the extreme Reissner-Nordstrøm black hole); right, $p$ odd. The different regions I and II are indicated.

\subsubsection{Non-dilatonic branes.}

As discussed in [12], the general brane solution case (2.10) can be divided in two classes: $p$ odd or $p$ even.

Let us first consider the $p$ odd case. In the region $r>0$, the exterior region, the function $A(r)$ is analytic and positive and vanishes as $r \rightarrow 0$. If we take $A$ to be our new radial variable instead of $r$, we see that $A$ can be continued through the horizon to negative $A$ [12]. 
The range of $A$ is from -1 to 1 . The analytic extension of the metric is

$$
\mathrm{d} s^{2}=A^{2} \mathrm{~d} x_{\mu} \mathrm{d} x^{\mu}+\left(1-A^{p+1}\right)^{-2 / \kappa}\left[w^{2}\left(1-A^{p+1}\right)^{-2} A^{-2} \mathrm{~d} A^{2}+\mathrm{d} \Omega^{2}\right],
$$

which is even in $A$. This leads to

$$
F(A)=-\frac{w}{\kappa}(\operatorname{sign} A)\left[B_{A^{p+1}}\left(\frac{-1}{p+1}, 1-\frac{2}{\kappa}\right)+2 B_{A^{p+1}}\left(\frac{p}{p+1},-\frac{2}{\kappa}\right)\right] .
$$

The embedding functions (2.8) are then odd in $A$. This means that the embedded space is symmetric around the horizon and completely non-singular. (Up to a factor, $A$ corresponds to $\omega$ in [12], where the same continuation was discussed.) The Penrose diagram for these $p$ odd brane geometries is shown in figure 3. For the non-dilatonic branes, D3 and M5 fit this picture. The embedding (figure (4) shows these features nicely. It is clearly visible that there is no interior region, just two symmetric 'exterior' regions connected in the AdS throat as was expected from the Penrose diagram.

In the $p$ even case, the metric and embedding functions are neither even nor odd. It is useful in this case to adopt so-called Schwarzschild coordinates, which are defined by $R^{\kappa}=r^{\kappa}+1$. In these coordinates the horizon (which is still a coordinate singularity) is at $R=1$. At $R=0$ there is a true curvature singularity (the radial variable used in the Reissner-Nordstrøm example actually was a Schwarzschild variable). Expressed in this coordinate, $A(R)$ can be continued through the horizon into negative $A$ and its range is $\{-\infty, 1\}$. As already stated in [12], the Penrose diagram for these spaces is equivalent to the extreme Reissner-Nordstrøm diagram (see figure 3).

The embedding of the $M 2$-brane metric illustrates these features (figure 5). The expression (2.14) of $F$ is well defined only in the region $R>1$. It is not possible to find a continuous expression for $F$ valid in both regions $(0<R \leq 1$ and $R>1)$. Nevertheless, a continuous embedding is obtained using in the interior region

$$
F(R)=\frac{w}{\kappa}\left[B_{R^{\kappa}}\left(\frac{1}{p+1}+\frac{2}{\kappa},-\frac{1}{p+1}\right)-2 B_{R^{\kappa}}\left(\frac{1}{p+1}+\frac{3}{\kappa},-\frac{1}{p+1}\right)\right] .
$$

Note that the global structure of the $M 2$ indeed resembles that of the extreme RN black hole (cf figures 5 and 2).

As in the RN case, we can directly read off some of the global properties from the figures. Again the spaces admit closed timelike curves or, as it is put in [2], they are wrapped in time. The alternative is of course again taking the covering space, resulting in an infinite stack of connected universes. 

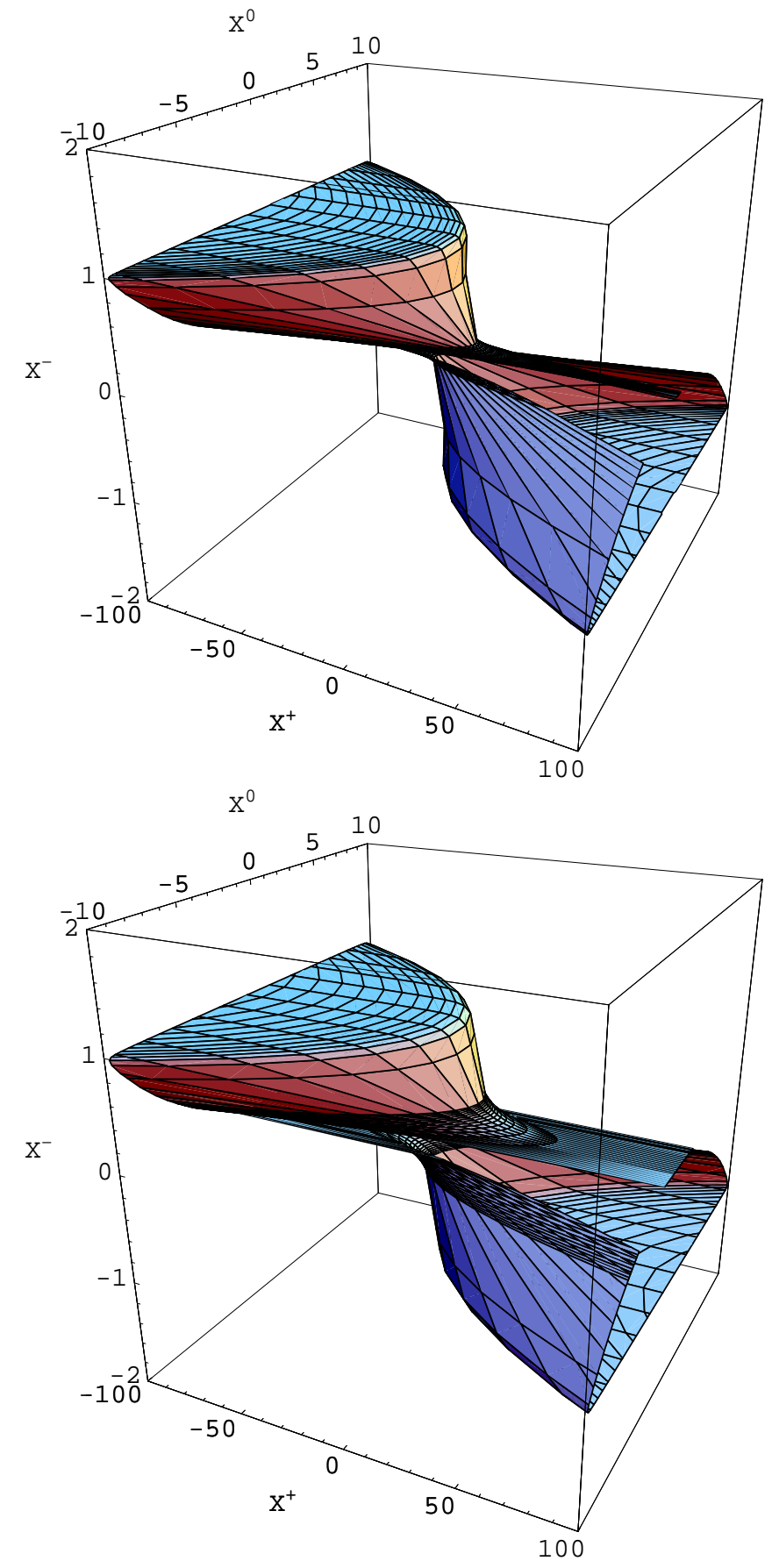

Figure 4: $p$ odd branes. a: (top) D3 and b: (bottom) M5. Two asymptotically flat regions connected in AdS. 


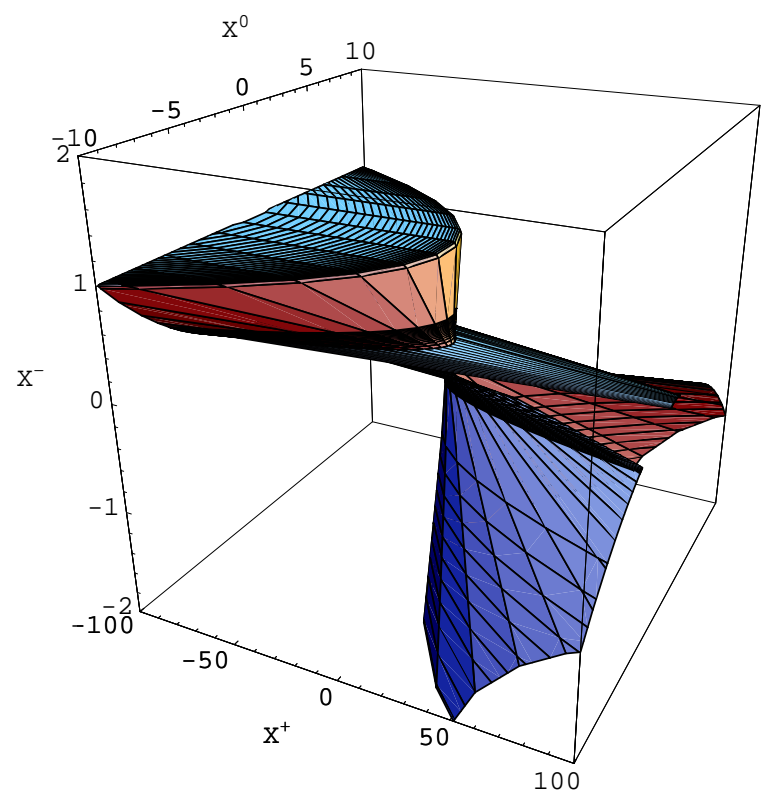

Figure 5: $p$ even: M2. It has the same structure as the $R N$ black hole

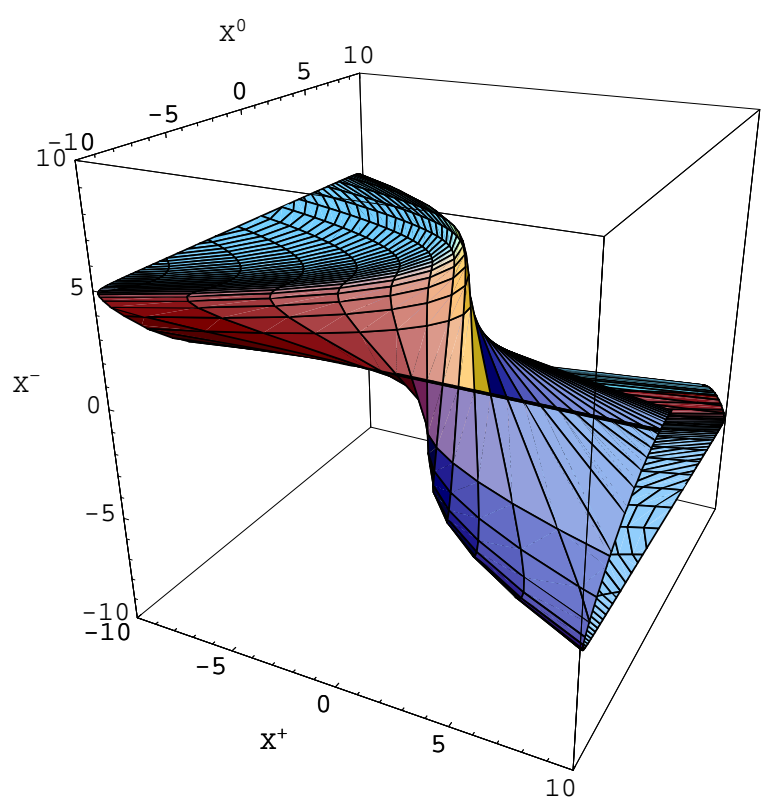

Figure 6: M5-brane metric parametrized by $r$ and $v=t+R^{*}$. Constant $v$ lines (infalling lightlike geodesics) pass the horizon into the next region. 
To show that the horizon is completely regular, we can reparametrize the embedding in Finkelstein coordinates as in the case of the RN black hole. Figure 6 shows part of the embedding of the M5-brane metric using these Finkelstein coordinates. The constant $v$ lines ( $v$ is the advanced Finkelstein time coordinate $v=t+R^{*}$ as defined in most textbooks) correspond to infalling lightlike geodesics and they clearly pass through the horizon into the next region.

\section{The brane action}

We would like to write the action of a brane placed in the background of other branes using the embedding in the $D+2=n+p+3$ spacetime. A typical (schematic) form of the action is

$$
\begin{aligned}
S_{p+1} & =\int_{W} \mathrm{~d}^{p+1} \xi \sqrt{-\operatorname{det} \mathcal{G}_{\mu \nu}}+\int_{B(W=\partial B)} \Omega_{(p+2)} \\
& +\int_{W} \mathrm{~d}^{p+1} \xi\left[\lambda_{1} \phi_{1}\left(X^{+}, X^{-}, X^{\mu}\right)+\lambda_{2} \phi_{2}\left(X^{-}, X^{\alpha}\right)\right],
\end{aligned}
$$

where $W$ is the $(p+1)$-dimensional worldvolume of the brane. The expression for $\mathcal{G}_{\mu \nu}$ differs for each case. For example, for $D p$-branes $\mathcal{G}_{\mu \nu} \equiv \partial_{\mu} X^{M} \partial_{\nu} X^{N} \eta_{M N}+\mathcal{F}_{\mu \nu}$, with $\mathcal{F}_{\mu \nu}$ the field strength of the gauge field living on the worldvolume of the brane. The fields $\lambda_{1}, \lambda_{2}$ are two Lagrange multipliers implementing the constraints (2.9). $\Omega_{(p+2)}\left(X^{M}\right)$ is a function of the forms coupling to the brane, such that it reduces to the appropriate Wess-Zumino term when projected on the physical hypersurface determined by the constraints. The explicit form of $\Omega_{(p+2)}$ will be determined, for the non-dilatonic D3-, M2- and $M 5$-brane cases, in the next subsections.

\subsection{Embedding the field strength}

Let us now study the embedding in the $(D+2)$-dimensional space of the field strengths appearing in the Wess-Zumino term of the action (3.1). We will follow the suggestion of [3]. The idea would be that a brane (extended in $p$ spatial directions) fluctuating in a spacetime with two times should evolve in both the time directions, and therefore couple to a $(p+3)$-form field strength. We assume therefore that the $(D+2)$-dimensional theory can be coupled to rank $p+3$ electric field strengths $K_{e}$, and to rank $n$ magnetic field strengths $K_{m}$, coupling to branes with $p$ spacelike directions evolving in 2 times. We then ask that the rank $p+2$ field strengths $F$ coupled to $p$-branes in $D$-dimensional supergravity be a restriction of $K_{e}\left(K_{e}+K_{m}\right.$ for the $D 3$-brane case) to the $D$-dimensional hypersurface $\Sigma$. 
This ansatz is the most natural one for the $D 3$-brane, because in this case the 10-dimensional self-dual field strength is extended to a self-dual field strength in 12 dimensions. If there were a supergravity theory in $D=12$, the bosonic configuration with flat (10,2)-space and a constant self-dual field strength would solve the equations of motion. This is obvious for the Maxwell equation (there can be no Chern-Simons terms built from a 5-form potential in 12 dimensions and so the Maxwell equation would take the standard form), but for the Einstein equations it is only true because the field strength is self-dual. In a $D$-dimensional spacetime with Euclidean or Kleinian signature (i.e. zero or two times) a self-dual field strength has a vanishing energy-momentum tensor for $D=4 \bmod 4$. (For Lorentzian signature it is $D=2$ mod 4.) What this would mean is that the ten-dimensional $D 3$-brane solution would just be the projection to a complicated hypersurface of an almost trivial 12-dimensional supergravity solution.

Let us start by analysing how an electric $(p+2)$-form field strength $F^{(p+2)}$ coupling to a $p$-brane becomes embedded in the $(D+2)$-dimensional space. Our aim is to obtain $F$ as a restriction of a $(p+3)$-form $K^{(p+3)}$ to the $D$-dimensional hypersurface $\Sigma$.

A general non-dilatonic brane is described in $D$ dimensions by the fields [13]

$$
\begin{aligned}
\mathrm{d} s^{2} & =H^{-2 /(p+1)}\left[-\mathrm{d} t^{2}+\mathrm{d} x_{1}^{2}+\cdots+\mathrm{d} x_{p}^{2}\right]+H^{2 / \kappa}\left[\mathrm{d} r^{2}+r^{2} \mathrm{~d} \Omega_{D-p-2}^{2}\right], \\
G_{01 \ldots p} & =-H^{-1}=-A^{p+1}, \\
\Phi & =\text { constant }=0,
\end{aligned}
$$

with the notation of section 2. The only non-vanishing components of the field strength $F=\mathrm{d} G$, are those related to the components given above by antisymmetry. Taking that into account, we can write the (electric) field strength as

$$
F=-(p+1) A^{p} A^{\prime} \mathrm{d} r \wedge \mathrm{d} t \wedge \mathrm{d} x_{1} \wedge \cdots \wedge \mathrm{d} x_{p},
$$

where a prime denotes differentiation with respect to $r$.

To find the embedding, we start by considering a constant $(p+3)$-form in $D+2$ dimensions

$$
K_{e}=\frac{p+1}{(p+3) !} \epsilon_{\mu_{0}^{\prime} \ldots \mu_{p+2}^{\prime}} \mathrm{d} X^{\mu_{0}^{\prime}} \wedge \mathrm{d} X^{\mu_{1}^{\prime}} \wedge \cdots \wedge \mathrm{d} X^{\mu_{p+2}^{\prime}}
$$

Primed indices run over $\mu^{\prime}=0, \ldots, p+2$. In order to obtain a rank $(p+2)$ field strength, we contract $K_{e}$ with a vector field $V$, with components $V=V^{M}\left(\frac{\partial}{\partial X^{M}}\right)$, which we take to be arbitrary (there is a sign ambiguity in this contraction; we chose to make it on the left, i.e. $\left.A_{\mu_{1}^{\prime} \ldots \mu_{p+2}^{\prime}}^{\prime}=V^{\mu_{0}^{\prime}} K_{\mu_{0}^{\prime} \ldots \mu_{p+2}^{\prime}}\right)$. Such a contraction yields

$$
K_{e}(V)=\frac{p+1}{(p+2) !} \epsilon_{\mu_{0}^{\prime} \ldots \mu_{p+2}^{\prime}} V^{\mu_{0}^{\prime}} \mathrm{d} X^{\mu_{1}^{\prime}} \wedge \cdots \wedge \mathrm{d} X^{\mu_{p+2}^{\prime}}
$$


Then, we reduce the resulting $(p+2)$-form to the $D$-dimensional hypersurface by using the embedding functions (2.8),

$$
\begin{aligned}
\left.K_{e}(V)\right|_{\Sigma}= & \frac{1}{2}(p+1) A^{\prime} A^{p+1} \mathrm{~d} r \wedge \mathrm{d} t \wedge \mathrm{d} x_{1} \wedge \cdots \wedge \mathrm{d} x_{p} \\
& \times \times\left[2 V^{\mu} x_{\mu}+V^{+}\left(\frac{F^{\prime}}{A^{\prime}}-x^{\mu} x_{\mu}\right)-V^{-}\right],
\end{aligned}
$$

where we have defined $V^{ \pm} \equiv V^{p+2} \pm V^{p+1}$. Next we impose that $\left.K_{e}(V)\right|_{\Sigma}=F$. To determine $V^{M}$, we use the ansatz $V^{M}=\left(V^{\mu^{\prime}}, V^{\alpha}\right)=\left(\alpha(r) X^{\mu^{\prime}}, V^{\alpha}\right)$. Because $K_{e}$ only has components in the longitudinal directions, $V^{\alpha}$ stays undetermined. When the field strength also includes a magnetic part, this $V^{\alpha}$ comes into play, as we will see in the next section. It follows that, in order for (3.6) to match with (3.3), $\alpha(r)$ has to obey

$$
\alpha(r)\left(\frac{A F^{\prime}}{A^{\prime}}-F\right)=-\frac{2}{A} .
$$

We use (2.7) to determine $\alpha(r)$

$$
\alpha(r)=\frac{2}{A F+w^{2} C^{2}\left(2 C^{\kappa}-1\right)} .
$$

The general form of the vector field in terms of the $(D+2)$-dimensional coordinates is

$$
V^{\mu^{\prime}}=\frac{2 X^{\mu^{\prime}}}{w^{2}\left(X^{\alpha} X_{\alpha}\right)\left[2\left(X^{\alpha} X_{\alpha}\right)^{\kappa / 2}-1+w^{-2}\right]-X^{M} X_{M}} .
$$

If we take the near-horizon limit, with use of (2.15) we find $\alpha(r) \sim 1 / w^{2}$, which matches with the results in [1] (see formula (2.17) in that paper).

\subsection{D3-brane embedding}

The 10-dimensional Wess-Zumino term is the integral of the self-dual field strength $F$ that couples to the D3-branes solution of the type IIB supergravity theory. For the 12-dimensional theory we construct a self-dual 6 -form $K$, i.e.

$$
\star K \wedge K=\eta_{12}|K|^{2},
$$

where $\eta_{12}$ is the volume form on the 12-dimensional spacetime. Our aim is to obtain $F$ as a restriction of $K$ to the 10 -dimensional surface $\Sigma$. The $D 3$-brane is described by the fields (3.2) introduced in the previous subsection, with $p+1=\kappa=4, D=10$. In this case, 
the only non-vanishing components of the field strength $F=\mathrm{d} G$ are those related to the components in (3.2) by either antisymmetry or self-duality. Taking that into account we can write the (anti)self-dual field strength as

$$
F=H^{\prime} H^{-2} \mathrm{~d} t \wedge \mathrm{d} x \wedge \mathrm{d} y \wedge \mathrm{d} z \wedge \mathrm{d} r+H^{\prime} H^{-1 / 2} \sqrt{|g|} \mathrm{d} \theta \wedge \mathrm{d} \phi_{1} \wedge \mathrm{d} \phi_{2} \wedge \mathrm{d} \phi_{3} \wedge \mathrm{d} \phi_{4},
$$

where a prime denotes differentiation with respect to $r$. In terms of the embedding functions, the last expression reads

$$
F=-4 A^{\prime} A^{3} \mathrm{~d} t \wedge \mathrm{d} x \wedge \mathrm{d} y \wedge \mathrm{d} z \wedge \mathrm{d} r+4 \omega_{(5)}
$$

where $\omega_{(5)} \equiv \sin (\theta)^{4} \sin \left(\phi_{1}\right)^{3} \sin \left(\phi_{2}\right)^{2} \sin \left(\phi_{3}\right) \mathrm{d} \theta \wedge \mathrm{d} \phi_{1} \wedge \cdots \wedge \mathrm{d} \phi_{4}$ is the volume form on the unit 5-sphere.

To find the embedding, we start by considering a constant self-dual six-form in 12 dimensions

$$
K=\frac{4}{6 !}\left(\epsilon_{\mu_{0}^{\prime} \ldots \mu_{5}^{\prime}} \mathrm{d} X^{\mu_{0}^{\prime}} \wedge \mathrm{d} X^{\mu_{1}^{\prime}} \wedge \cdots \wedge \mathrm{d} X^{\mu_{5}^{\prime}}+\epsilon_{\alpha_{1} \ldots \alpha_{6}} \mathrm{~d} X^{\alpha_{1}} \wedge \mathrm{d} X^{\alpha_{1}} \wedge \cdots \wedge \mathrm{d} X^{\alpha_{6}}\right) .
$$

We have written $K$ in a manifestly $S O(4,2) \times S O(6)$ invariant form. In order to obtain a rank-5 field strength, we contract, as we have done for the general electric case, $K$ with a vector field $V$, with components $V=V^{M}\left(\frac{\partial}{\partial X^{M}}\right)$. Such a contraction yields

$$
K(V)=\frac{4}{5 !}\left(\epsilon_{\mu_{0}^{\prime} \ldots \mu_{5}^{\prime}} V^{\mu_{0}^{\prime}} \mathrm{d} X^{\mu_{1}^{\prime}} \wedge \cdots \wedge \mathrm{d} X^{\mu_{5}^{\prime}}+\epsilon_{\alpha_{1} \ldots \alpha_{6}} V^{\alpha_{1}} \mathrm{~d} X^{\alpha_{2}} \wedge \cdots \wedge \mathrm{d} X^{\alpha_{6}}\right) .
$$

Then, we reduce the resulting 5-form to the 10-dimensional hypersurface by using the embedding functions (2.8). By requiring the matching $\left.K(V)\right|_{\Sigma}=F$, we obtain the constraints for our vector field $V$. The resulting 5 -form $K(V)$ is the looked-for Wess-Zumino form $\Omega_{5}$.

Let us analyse separately the two terms in the right-hand side of (3.12)-(3.14). The electric part has already been studied in the general case in the previous subsection. In this case it gives $V^{\mu^{\prime}}=\alpha(r) X^{\mu^{\prime}}$ with

$$
\alpha(r)=\frac{2}{2\left(X^{\alpha} X_{\alpha}\right)^{3}-X^{M} X_{M}} .
$$

The angular part in (3.13) can be rewritten in terms of the radial coordinate $r$ and of the angular coordinates $\theta, \phi_{i}(i=1, \ldots, 4)$ as

$$
\begin{aligned}
\frac{1}{6 !} \epsilon_{\alpha_{1} \ldots \alpha_{6}} \mathrm{~d} X^{\alpha_{1}} \wedge \cdots \wedge \mathrm{d} X^{\alpha_{6}} & =C^{\prime} C^{5} \sqrt{g_{S_{5}}} \mathrm{~d} r \wedge \mathrm{d} \theta \wedge \mathrm{d} \phi_{1} \wedge \cdots \wedge \mathrm{d} \phi_{4} \\
& =C^{\prime} C^{5} \mathrm{~d} r \wedge \omega_{5},
\end{aligned}
$$


where $g_{S_{5}}$ is the determinant of the metric of the unit 5-sphere in polar coordinates.. In order for the second term in (3.14) to match with the second term in (3.12), we have to require that the vector $V$ points in the radial direction when decomposed on the $r, \theta, \phi_{i}$ basis, that is

$$
V^{\alpha} \frac{\partial}{\partial X^{\alpha}} \equiv V^{\alpha} \frac{\partial r}{\partial X^{\alpha}} \frac{\partial}{\partial r}
$$

This gives

$$
\frac{1}{5 !} \epsilon_{\alpha_{1} \ldots \alpha_{6}} V^{\alpha_{1}} \mathrm{~d} X^{\alpha_{2}} \wedge \cdots \wedge \mathrm{d} X^{\alpha_{6}}=C^{\prime} C^{5} V^{\alpha} \frac{\partial r}{\partial X^{\alpha}} \omega_{5}
$$

The matching with 3.12 requires

$$
V^{\alpha} \frac{\partial r}{\partial X^{\alpha}}=\left(C^{\prime} C^{5}\right)^{-1}
$$

which is solved, with the ansatz $V^{\alpha}=\epsilon(r) X^{\alpha}$, by

$$
V^{\alpha}=C^{-6} X^{\alpha}=\left(1+r^{4}\right)^{-3 / 2} X^{\alpha} .
$$

We notice that $\epsilon(r=0)=\alpha(r=0)=1$, so that in the near-horizon approximation we have $V^{M}=X^{M}$ and $K(V)$ becomes the potential for the 12-dimensional self-dual form $K$ (up to a constant), making contact with the work in [四]. The general form of the vector field in terms of the 12-dimensional coordinates is

$$
V^{\mu^{\prime}}=\frac{2 X^{\mu^{\prime}}}{2\left(X^{\alpha} X_{\alpha}\right)^{3}-X^{M} X_{M}}, \quad V^{\alpha}=\frac{X^{\alpha}}{\left(X^{\beta} X_{\beta}\right)^{3}} .
$$

\subsection{M2-brane embedding}

For $M 2$ the Wess-Zumino form is the rank 4 (electric) field strength $F$. We require that this field strength be a restriction of $K_{e}$ to the 11-dimensional hypersurface.

The fundamental $M 2$-brane is described in 11 dimensions by the fields (3.2) with $p+1=3$, $\kappa=6$, and $F=\mathrm{d} G$ an electric field strength, i.e.

$$
F=-3 A^{\prime} A^{2} \mathrm{~d} x_{0} \wedge \mathrm{d} x_{1} \wedge \mathrm{d} x_{2} \wedge \mathrm{d} r .
$$

Repeating the general analysis, we require that $F=\left.K(V)\right|_{\Sigma}$, where $K$ is a constant 5-form in 13 dimensions

$$
K=\frac{3}{5 !} \epsilon_{\mu_{0}^{\prime} \ldots \mu_{4}^{\prime}} \mathrm{d} X^{\mu_{0}^{\prime}} \wedge \mathrm{d} X^{\mu_{1}^{\prime}} \wedge \cdots \wedge \mathrm{d} X^{\mu_{4}^{\prime}}
$$

\footnotetext{
${ }^{7}$ We have used the relation $C^{2}(r)=X^{\alpha} X_{\alpha}$, from which $\frac{\partial r}{\partial X^{\alpha}}=\frac{\partial r}{\partial C^{2}(r)} \frac{\partial C^{2}}{\partial X^{\alpha}}=\left(C C^{\prime}\right)^{-1} X^{\alpha}$.
} 
The vector field $V$, with components $V=V^{M}\left(\frac{\partial}{\partial X^{M}}\right)$, turns out to be $V^{M}=\left(V^{\mu^{\prime}}, V^{\alpha}\right)=$ $\left(\alpha(r) X^{\mu^{\prime}}, V^{\alpha}\right)$ where

$$
\alpha(r)=\frac{8 A}{4 A^{2} F+r^{2}\left(1+2 r^{6}\right)},
$$

and $V^{\alpha}$ stays undetermined. Written in terms of 13-dimensional coordinates, the general form of the vector field $V^{M}$ is

$$
V^{M}=\left(V^{\mu^{\prime}}, V^{\alpha}\right)=\left(\frac{8 X^{\mu^{\prime}}}{X^{\alpha} X_{\alpha}\left[3+2\left(X^{\alpha} X_{\alpha}\right)^{3}\right]-4 X^{M} X_{M}}, V^{\alpha}\right) .
$$

The $\Omega_{4}$ of (3.1) is $K(V)$.

\subsection{M5-brane embedding}

Let us now perform a similar construction for the $M 5$-brane background. We start with the 11-dimensional metric describing the geometry of the $M 5$-brane

$$
\mathrm{d} s^{2}=H^{-1 / 3}\left(-\mathrm{d} t^{2}+\mathrm{d} x_{1}^{2}+\cdots+\mathrm{d} x_{5}^{2}\right)+H^{2 / 3}\left(\mathrm{~d} r^{2}+r^{2} \mathrm{~d} \Omega_{(4)}\right),
$$

with $H=1+1 / r^{3}$. The $M 5$ is a solitonic solution of 11-dimensional supergravity [14, and is coupled in a magnetic way to the 4 -form

$$
F^{(4)}=\mathrm{d} A^{(3)}=H^{\prime} H^{-1 / 2} \sqrt{|g|} \mathrm{d} \theta \wedge \mathrm{d} \phi_{1} \wedge \cdots \wedge \mathrm{d} \phi_{3}=3 \omega_{(4)}
$$

and in an 'electric' way to the 7-form

$$
F^{(7)}=\star F^{(4)}+A^{(3)} \wedge F^{(4)}=-H^{-2} H^{\prime} \mathrm{d} x^{0} \wedge \cdots \wedge \mathrm{d} x^{5} \wedge \mathrm{d} r .
$$

The expression (3.28) for the electric coupling to $M 5$ has the same form (3.3) as the general electric field strength discussed previously, so we can apply the same procedure. Analogously to the $M 2$ case, we start from a constant 8-form $K^{(8)}$ in $d=13$ and contract it with an arbitrary vector (let us call it $V_{1}^{M}$ ) to a 7 -form

$$
K^{(8)}\left(V_{1}\right)=\frac{6}{7 !} \epsilon_{\mu_{0}^{\prime} \ldots \mu_{7}^{\prime}} V_{1}^{\mu_{0}^{\prime}} \mathrm{d} X^{\mu_{1}^{\prime}} \wedge \cdots \wedge \mathrm{d} X^{\mu_{7}^{\prime}}
$$

The requirement that $\left.K\left(V_{1}\right)\right|_{\Sigma}=F$ fixes the vector $V_{1}^{M}=\left(V_{1}^{\mu^{\prime}}, V_{1}^{\alpha}\right)$ to be of the form $V_{1}^{M}=\left(\alpha(r) X^{\mu^{\prime}}, V_{1}^{\alpha}\right)$ with

$$
\alpha(r)=\frac{2}{-X^{M} X_{M}+X^{\alpha} X_{\alpha}\left[8\left(X^{\beta} X_{\beta}\right)^{3 / 2}-3\right]} .
$$


Extra complications arise when we want to describe the $M 5$ propagating in the background of the antisymmetric gauge fields $F^{(4)}$ and $F^{(7)}$ of 11-dimensional supergravity. Indeed, the 3 -form field strength $\mathcal{H}$ [15] living on the worldvolume of the $M 5$ couples to the above fields giving the following expression for the Wess-Zumino term:

$$
\left.\Omega_{7}\right|_{\Sigma}=F^{(7)}-\frac{1}{2} \mathcal{H} \wedge F^{(4)}
$$

It is therefore this last expression that has to be embedded in the 13-dimensional space. We will follow and generalize the discussion in [1], where an analogous treatment of the M5 Wess-Zumino term has been given for the near-horizon case.

As for the near-horizon limit, we only need the embedding of the fields $F^{(7)}$ and $F^{(4)}, \mathcal{H}$ being a field living on the physical worldvolume of the brane, only defined over $\Sigma$.

To find the embedding of $F^{(4)}$ we can proceed in a way completely similar to the magnetic part of the D3-brane field strength: we start from a constant 5-form in 13 dimensions,

$$
K^{(5)}=\star K^{(8)}=\frac{6}{5 !} \epsilon_{\alpha_{1} \ldots \alpha_{5}} \mathrm{~d} X^{\alpha_{1}} \wedge \mathrm{d} X^{\alpha_{1}} \wedge \cdots \wedge \mathrm{d} X^{\alpha_{5}} .
$$

In order to obtain a rank-4 field strength we contract, as we have done for the other cases, $K$ with a vector field $V_{2}$, with components $V_{2}=V_{2}^{M}\left(\frac{\partial}{\partial X^{M}}\right)$. Such a contraction yields

$$
K^{(5)}\left(V_{2}\right)=\frac{6}{4 !} \epsilon_{\alpha_{1} \ldots \alpha_{5}} V_{2}^{\alpha_{1}} \mathrm{~d} X^{\alpha_{2}} \wedge \cdots \wedge \mathrm{d} X^{\alpha_{5}} .
$$

Then we reduce the resulting 4-form to the 11-dimensional hypersurface by using the embedding functions (2.8)

$$
\left.K^{(5)}\left(V_{2}\right)\right|_{\Sigma}=C^{\prime} C^{4} V_{2}^{\alpha} \frac{\partial r}{\partial X^{\alpha}} \omega_{4}
$$

By requiring $\left.K^{(5)}\left(V_{2}\right)\right|_{\Sigma}=F^{(4)}$, we can solve for the vector field $V_{2}$, which turns out to be (following the same arguments as for D3) $V_{2}^{M}=\left(V_{2}^{\mu^{\prime}}, V_{2}^{\alpha}\right)$, with $V_{2}^{\mu^{\prime}}$ free and

$$
V_{2}^{\alpha}=C^{-5} X^{\alpha}
$$

Note that the two vectors $V_{1}^{M}$ and $V_{2}^{M}$ have constrained components on orthogonal subspaces ( $V_{1}$ on the indices $\mu^{\prime}$ and $V_{2}$ on the indices $\alpha$ ), so we can use for the projection the single vector $V^{M}=\left(V_{1}^{\mu^{\prime}}, V_{2}^{\alpha}\right)$.

We then find, analogously to [1], that the 7-form appearing in the embedded WessZumino term has the following form:

$$
\Omega_{7}=K^{(8)}(V)-\frac{1}{2} \mathcal{H} \wedge K^{(5)}(V),
$$

which, restricted to the hypersurface $\Sigma$, reduces to the closed form (3.31). 


\section{Particles in a brane background}

Now we would like to analyse the behaviour of a probe particle put in a $p$-brane background. In particular, we would like to see whether the above construction (an embedding in a higher-dimensional flat space) can shed new light on the dynamics of the system.

We can interpret the particle in a background as a constrained Hamiltonian system and apply to it the standard rules of constrained quantization. In particular, the constraints (2.9) selecting the physical hypersurface can be seen to appear in that context as secondclass primary constraints.

Recently, however, an approach has been developed by Bars and collaborators [6] for studying Hamiltonian systems embedded in higher-dimensional spaces with two times. Here the constraints follow from an $S p(2)$ gauge symmetry internal to the particle system?. They are therefore first-class constraints (instead of second class). In that way it was shown that any physical system that can be written as a gauge-fixed form of a particular $(D+2)$ dimensional action with $S p(2)$ gauge symmetry (the action (2) in [6] (BDA action)) has, in fact, a (maybe hidden) $S O(D, 2)$ off-shell symmetry.

Having found an embedding of brane backgrounds in a flat spacetime with two time directions, it is natural to wonder whether a system given by a particle in a brane background could be written as a gauge-fixed form of the BDA action (and has therefore, if this were the case, a hidden $S O(D, 2)$ invariance).

It turns out that, in general, this is not true: the action for a particle in a brane background can be written as gauge-fixed form of the BDA action only when the background metric is conformally flat, that is only in the asymptotic regions of the background. The details of our application of the construction in [6] are given in section 4.2. Before doing this, let us describe how the resolution of the constraints works when using the second-class constraints approach.

\subsection{Hypersurface as a set of second-class constraints}

We start from a free Hamiltonian in the embedding space,

$$
H_{0}=P^{M} P_{M}=-4 P_{+} P_{-}+P_{\mu}^{2}+P_{\alpha}^{2}
$$

on which we impose the following two constraints:

$$
\begin{aligned}
& \phi_{1}=-X^{+} X^{-}+\left(X^{\mu}\right)^{2}+X^{-} F\left(X^{-}\right), \\
& \phi_{2}=\left(X^{\alpha}\right)^{2}-C\left(X^{-}\right)^{2},
\end{aligned}
$$

\footnotetext{
${ }^{8}$ It is a local version of the $S p(2)$ global group relating coordinates and conjugate momenta in phase space.
} 
to constrict the motion of the particle to the hypersurface (note that $\eta_{+-}=-\frac{1}{2}, \eta^{+-}=-2$ ). Note that $F\left(X^{-}\right)$should be interpreted as $F\left(\bar{A}\left(X^{-}\right)\right)$. Following the general procedure of quantizing constrained Hamiltonian systems, we add these primary constraints to the Hamiltonian,

$$
H_{*}=H_{0}+u^{i} \phi_{i}
$$

In order to find out whether there are any secondary constraints, we calculate the bracket of the primary constraints with the Hamiltonian,

$$
\dot{\phi}_{i}=\left\{\phi_{i}, H_{*}\right\} \approx\left\{\phi_{i}, H_{0}\right\}+u^{j}\left\{\phi_{i}, \phi_{j}\right\}
$$

the brackets are defined by $\left\{X^{M}, P_{N}\right\}=\delta^{M}{ }_{N}$. From this we find the following two secondary constraints:

$$
\begin{aligned}
& \chi_{1}=X^{\mu^{\prime}} P_{\mu^{\prime}}-\left(X^{-} F\right)^{\prime} P_{+}, \\
& \chi_{2}=X^{\alpha} P_{\alpha}+\left(C^{2}\right)^{\prime} P_{+},
\end{aligned}
$$

where a prime denotes differentiation with respect to $X^{-}$. Taking the bracket of the new constraints with the Hamiltonian $H_{*}$ we find two equations that allow us to fix the coefficients $u^{i}$ : there are no tertiary constraints.

These four constraints are all what is called second class, because there is no subset of them that has zero bracket with all other constraints. In order to deal with them we make use of the Dirac analysis [16]. Let us introduce the so-called Dirac brackets

$$
\{f, g\}_{D}=\{f, g\}-\left\{f, \varphi_{a}\right\} C^{a b}\left\{\varphi_{b}, g\right\},
$$

where $\varphi_{a}=\left(\phi_{i}, \chi_{i}\right), a=1, \ldots, 4$ and $C^{a b}$ is the inverse of the matrix

$$
\Delta_{a b}=\left\{\varphi_{a}, \varphi_{b}\right\}
$$

which is non-singular because all constraints are second class.

With these new brackets we can calculate the equations of motion via Hamilton's equations

$$
\dot{X}^{M}=\left\{X^{M}, H_{0}\right\}_{D}, \quad \dot{P}_{M}=\left\{P_{M}, H_{0}\right\}_{D} .
$$

Applying this to our case we find some very non-trivial differential equations for the $X^{M}$, describing the motion of a particle confined to the embedded spacetime,

$$
\begin{gathered}
\frac{1}{2} \ddot{X}_{M}=\dot{P}_{M}=\frac{1}{2 C^{2}\left(X^{-}\right)^{2}\left[\left(C^{\prime}\right)^{2}-F^{\prime}\right]} \times\left\{\left[2 \delta_{M}^{\mu^{\prime}} X_{\mu^{\prime}}+\delta_{M}^{-}\left(X^{-} F\right)^{\prime}\right] \times\right. \\
\left\{2 C^{2}\left[P^{\nu^{\prime}} P_{\nu^{\prime}}+\frac{1}{2}\left(P^{-}\right)^{2}\left(X^{-} F\right)^{\prime \prime}\right]+X^{-}\left(C^{2}\right)^{\prime}\left[P^{\beta} P_{\beta}-\frac{1}{2}\left(P^{-}\right)^{2}\left(C^{2}\right)^{\prime \prime}\right]\right\} \\
+\left[2 \delta_{M}^{\alpha} X_{\alpha}-\delta_{M}^{-}\left(C^{2}\right)^{\prime}\right] \times\left\{X^{-}\left(C^{2}\right)^{\prime}\left[P^{\nu^{\prime}} P_{\nu^{\prime}}+\frac{1}{2}\left(P^{-}\right)^{2}\left(X^{-} F\right)^{\prime \prime}\right]\right. \\
\left.\left.+2\left(X^{-}\right)^{2} F^{\prime}\left[P^{\beta} P_{\beta}-\frac{1}{2}\left(P^{-}\right)^{2}\left(C^{2}\right)^{\prime \prime}\right]\right\}\right\} .
\end{gathered}
$$


In the near-horizon limit we have $C^{2} \sim 1$ and $X^{-} F \sim w^{2}$. The equations of motion then take the much simpler form

$$
\frac{1}{2} \ddot{X}_{M}=\dot{P}_{M}=\frac{2}{w^{2}} \delta_{M}^{\mu^{\prime}} X_{\mu^{\prime}}\left(P^{\nu^{\prime}} P_{\nu^{\prime}}\right)-2 \delta_{M}^{\alpha} X_{\alpha}\left(P^{\beta} P_{\beta}\right)
$$

\subsection{First-class constraint approach}

We refer, for the context discussed here, to the recent series of papers by Bars and collaborators [6, 7]. There, many systems are discussed where the apparent symmetry of the action can be enlarged to a bigger, nonlinearly realized, $S O(D, 2)$ hidden symmetry with explicit time-dependent generators. They show that all these (very different) actions can be written as one and the same action in $D+2$ dimensions, with $(D, 2)$ signature, where the $S O(D, 2)$ is now realized linearly. In that context, the different actions can be seen as following from different gauge choices of a hidden $S p(2)$ gauge symmetry present in the $(D+2)$-dimensional action. In some sense, the point of view in [6, 7] is that every time that there is a hidden $S O(D, 2)$ off-shell symmetry in an action, this should indicate that there is an underlying two-times physical spacetime controlling the system. The action considered, is just one of a collection of actions all related by some sort of duality, and all derivable by the same underlying higher-dimensional theory through a particular gauge choice.

A nice point is that, in particular, the constraints that define the embedding of the $A d S_{5} \times S_{5}$ in 12 dimensions can be seen as originating from particular choices of the $S p(2)$ gauge on the 12-dimensional action. This suggests that the same could be extended to our case, just with more general constraints. We were, therefore, led to investigate whether that scenario also fits in the non-near-horizon case.

Let us consider the BDA action [6]

$$
S_{0}=\int_{0}^{\tau} \mathrm{d} \tau\left(D_{\tau} X_{i}^{M}\right) \epsilon^{i j} X_{j}^{N} \eta_{M N}
$$

where $i, j=1,2 \in S p(2, R)$,

$$
\begin{aligned}
& X_{1}^{M}=X^{M}, \quad X_{2}^{M}=P^{M}=\frac{\partial S_{0}}{\partial \dot{X}^{N}} \eta^{M N} \\
& D_{\tau} X_{i}=\frac{\mathrm{d} X_{i}}{\mathrm{~d} \tau}-\frac{1}{2} A_{i}^{j} X_{j} \\
& A_{i}^{j}(\tau) \in \operatorname{Adj} S p(2, R) .
\end{aligned}
$$

It can be rewritten as

$$
S_{0}=\int_{0}^{\tau} \mathrm{d} \tau\left(\partial_{\tau} X^{M} P^{N}-\frac{1}{2} X_{i}^{M} \epsilon^{i j} A_{j}{ }^{k} X_{k}^{N}\right) \eta_{M N} .
$$


We want to see whether, with an appropriate choice of $S p(2, R)$ gauge, it reduces to the $D$-dimensional action describing the motion of a particle in a background generated by a brane

$$
S=\int_{0}^{\tau} \mathrm{d} \tau e^{-1} G_{m n} \frac{\mathrm{d} y^{m}}{\mathrm{~d} \tau} \frac{\mathrm{d} y^{n}}{\mathrm{~d} \tau}
$$

where

$$
\begin{aligned}
y^{m} & =\left(x^{\mu}, r, n^{\alpha}\right), \quad n^{\alpha} n_{\alpha}=1, \\
\mathrm{~d} s^{2} & \equiv G_{m n} \mathrm{~d} y^{m} \mathrm{~d} y^{n}=A^{2}(r) \mathrm{d} x^{\mu} \mathrm{d} x_{\mu}+B^{2}(r) \mathrm{d} r^{2}+C^{2}(r) \mathrm{d} \Omega_{n-1}^{2},
\end{aligned}
$$

and $e^{-1}$ is the worldline einbein.

To this end, we make use of the embedding (2.8), that is

$$
X^{M}=\left(A(r), F(r)+A(r) x^{\mu} x_{\mu}, A(r) x^{\mu}, C(r) n^{\alpha}\right) .
$$

By requiring the action (4.14) to be invariant for variations with respect to the functions $A_{i}{ }^{j}$, we obtain the three constraints: $X^{M} X_{M}=0, X^{M} P_{M}=0, P^{M} P_{M}=0$.

The constraint $X^{M} X_{M}=0$ gives

$$
A(r) F(r)=C^{2}(r)
$$

From (2.7) and (4.18), we can eliminate $F(r)$ and obtain

$$
B^{2}=C^{2}-F^{\prime} A^{\prime}=\left(C^{\prime}-\frac{C}{A} A^{\prime}\right)^{2}
$$

Note this new condition relating the 3 functions $A, B, C$ appearing in the metric.

Now impose the constraint $X^{M} P_{M}=0$, with

$$
P_{M}=\left(0, P_{+}, \frac{p_{\mu}}{A(r)}, p_{\alpha}\right)
$$

which gives

$$
P_{+}=\frac{2}{A(r)}\left(p_{\mu} x^{\mu}+C(r) n^{\alpha} p_{\alpha}\right)
$$

Let us now substitute the gauge-fixed expressions for $X^{M}$ and $P^{M}$ into the action (4.14). We obtain

$$
S_{0}=\int_{0}^{\tau} \mathrm{d} \tau\left[\frac{\mathrm{d} x^{\mu}}{\mathrm{d} \tau} p_{\mu}+\left(\dot{C}-\frac{C}{A} \dot{A}\right) n^{\alpha} p_{\alpha}+C \frac{\mathrm{d} n^{\alpha}}{\mathrm{d} \tau} p_{\alpha}-\frac{1}{2} A_{22}\left(\frac{p^{\mu} p_{\mu}}{A^{2}}+p_{\alpha} p^{\alpha}\right)\right],
$$


where, for all functions $f(r), \dot{f}=f^{\prime} \frac{d r}{d \tau}$. Then, after eliminating $p_{\mu}, p_{\alpha}$ through the equations of motion, we obtain

$$
S_{0} \rightarrow \frac{1}{2} \int_{0}^{\tau} \mathrm{d} \tau \frac{1}{A_{22}}\left[A^{2}(r) \frac{\mathrm{d} x_{\mu}}{\mathrm{d} \tau} \frac{\mathrm{d} x^{\mu}}{\mathrm{d} \tau}+B^{2}(r) \dot{r}^{2}+C^{2}(r) \frac{\mathrm{d} n_{\alpha}}{\mathrm{d} \tau} \frac{\mathrm{d} n^{\alpha}}{\mathrm{d} \tau}\right],
$$

where $B$ is given by (4.19). This action has the form (4.15).

In this way we have seen that, under condition (4.19), the action for a particle in a brane background has a hidden $S O(D, 2)$ symmetry. We have then found that the BDA action (4.14) reduces, by appropriate gauge fixings, to the action (4.15) not for the general brane background metric (2.1) but only when the constraint (4.19) is satisfied.

This condition is fulfilled for an $A d S \times S$ metric with equal radii for the two factors, but not for a general brane background, with $A, B, C$ given by the usual harmonic functions.

In particular, this is true for the near-horizon limit of $D 3$, but not for $M 2$ and $M 5$. In fact, condition (4.19) follows precisely by imposing the metric (2.1) to be conformally flat. Starting from (2.1) and using variable $C / A$, the metric takes the form

$$
\mathrm{d} s^{2}=A(r)^{2} \mathrm{~d} x^{\mu} \mathrm{d} x_{\mu}+\frac{B(r)^{2}}{\left(C^{\prime}-\frac{C}{A} A^{\prime}\right)^{2}} A(r)^{2} \mathrm{~d}\left(\frac{C}{A}\right)^{2}+A(r)^{2}\left(\frac{C}{A}\right)^{2} \mathrm{~d} \Omega_{n-1}^{2},
$$

which is conformally flat when (4.19) is satisfied.

\section{Discussion}

Our aim in this paper has been to develop a global description of the spacetime geometries of $M$ - and $D$-branes by isometrically embedding them in flat spacetimes with two extra dimensions and two times, thus extending the ideas of [1]. We have gained a rather clear global picture of the geometry, giving an insight into the structure around coordinate singularities and in the symmetries. In particular, the differences between $p$-branes with $p$ even and $p$ odd, previously pointed out in [12], are clearly apparent. Like the familiar embedding of anti-de Sitter spacetime as a quadric, our embeddings are periodic in time. This is consistent with some suggestions in [2], but one may of course always pass to a covering spacetime. But see [17] for an isometric embedding of the universal covering spacetime of $A d S_{2}$ in three and four-dimensional Minkowski spacetimes.

In the context of supergravity and string theory, $p$-branes are coupled to $(p+2)$-form field strengths. An embedding of the brane thus has to include, besides the embedding of the geometry, a prescription for the forms in the higher-dimensional space. This is obtained by defining constant $(p+3)$-forms in $D+2$ dimensions, and contracting them using a 
vector $V$. The form of $V$ is determined by matching the projection on the surface with the known forms for D3, M2 and M5. Unfortunately, the geometric significance of the vector field $V$, remains unclear. In the case of an $M 2$-brane it is not even unique, since the $V^{\alpha}$ components are arbitrary. A co-dimension-2 surface has a 2-dimensional normal plane. In the $D 3$ and $M 5$ cases, the vector $V$ does not lie in this 2-plane, except in the near-horizon limit. Specifically, the normal 2-plane is spanned by $\partial_{\mu} \phi_{1}$ and $\partial_{\mu} \phi_{2}$. One may check that $V$ is not a linear combination of $\partial_{\mu} \phi_{1}$ and $\partial_{\mu} \phi_{2}$. The bosonic action for probe branes in the embedded background (3.1) is completely determined after the construction of $V$.

One motivation for our work was the possibility of using this approach to quantize strings moving in these backgrounds. This we have not done, but we have indicated how Dirac's theory of constrained systems could, in principle, be used to quantize a point particle. One point of interest is that this would automatically build in the periodic temporal boundary conditions of [2] showing at least that the suggestion is mathematically consistent. Our work also makes contact with recent ideas on physics with two times. In particular, we see difficulties in extending the methods of [6] from the vicinity of the throat to the entire spacetime.

Finally, it is possible that the methods developed in this paper may be applicable to scenarios in which one regards the universe as a brane embedded in a higher-dimensional spacetime.

\section{Acknowledgments}

We would like to thank Renata Kallosh with whom GWG and AVP had stimulating discussions at the early stages of this work. We would also like to thank Paul Townsend and Walter Troost for discussions. This work is supported by the European Commission TMR programme ERBFMRX-CT96-0045. CH is supported by FCT (Portugal) through grant no PRAXIS XXI/BD/13384/97.

\section{References}

[1] P. Claus, R. Kallosh, J. Kumar, P.K. Townsend and A. Van Proeyen, JHEP06 (1998), $004 ;$ hep-th/9801206.

[2] G.W. Gibbons, Wrapping Branes in Space and Time, hep-th/9803206;

G.W. Gibbons, Anti-de-Sitter spacetime and its uses, in Mathematical and Quantum 
Aspects of Relativity and Cosmology, Proceedings of the 2nd Samos Meeting on Cosmology, Geometry and Relativity, S. Cotsakis and G.W. Gibbons eds, Lecture Notes in Physics Springer-Verlag, Berlin (2000)

[3] S.F. Hewson, An approach to F-theory, Nucl. Phys. B534 (1998) 513, hep-th/9712017.

[4] L.P. Eisenhart, Riemannian Geometry, Princeton university press Princeton (N.J.) (1997) $306 \mathrm{p}$.

[5] R. Penrose, A remarkable property of plane waves in General Relativity, Rev. Mod. Phys. vol. 37, N. 1 (1965) 215.

[6] I. Bars, C. Deliduman and O. Andreev, Gauged Duality, Conformal Symmetry, and Spacetime with Two Times, Phys. Rev. D58 (1998) 066004, hep-th/9803188.

[7] I. Bars, Hidden Symmetries, AdS $S_{D} \times S^{n}$, and the lifting of one-time-physics to twotime-physics, Phys. Rev. D59 (1999) 045019, hep-th/9810025.

[8] H.Goenner, Local Isometric embeddings of Riemannian Manifolds and Einstein's Theory of Gravitation, in "General Relativity and Gravitation: One hundred years after the birth of Einstein", Edited by A.Held, Vol.1, Plenum Press, 1980.

[9] A.Friedman, Isometric Embeddings of Riemannian manifolds into Euclidean spaces, Rev. Mod. Phys. 37 (1965) 201.

[10] G.Gibbons, P.Townsend, Vacuum interpolation in supergravity via super p-branes, Phys. Rev. Lett. 71 (1993) 3754; hep-th/9307049.

[11] J.Rosen, Embedding various relativistic Riemannian spaces in Pseudo-Euclidean spaces, Rev. Mod. Phys. 37 (1965) 204.

[12] G.W. Gibbons, G.T. Horowitz and P.K. Townsend, Higher-dimensional resolution of dilatonic black-hole singularities, Class. Quantum Grav. 12 (1995) 297, hep-th/9410073.

[13] M.J.Duff and J.X.Lu, The self-dual type IIB superthreebrane, Phys. Lett. B273 (1991) 409; for the M-branes see, for instance K.Stelle lectures on supergravity p-branes, hepth/9701088.

[14] M.J. Duff and K. Stelle, Phys. Lett. 253B (1991) 113; R. Güven, Phys. Lett. 276B (1992) 49 
[15] O. Aharony, String theory dualities from M-Theory, Nucl. Phys. B476 (1996) 470, hep-th/9604103; E. Bergshoeff, M. de Roo and T. Ortin, The Eleven-Dimensional FiveBrane, Phys. Lett. B386 (1996) 85, hep-th/9606118

[16] P.A,.M. Dirac, Lectures on quantum mechanics, Belfer Graduate School of Science, Yeshiva University, New York, 1964 -(Monographs series ; no. 2).

[17] E.L. Schucking and J-Z. Wang, The Two-dimensional Ivor, in Gravitation and Geometry, ed. W. Rindler and A. Trautman, Bibliopolis, Naples, 1987. 